Accepted By The Astrophysical Journal

Preprint typeset using $\mathrm{LATE}_{\mathrm{E}} \mathrm{X}$ style emulateapj v. 08/13/06

\title{
LIFETIME OF THE EMBEDDED PHASE OF LOW-MASS STAR FORMATION AND THE ENVELOPE DEPLETION RATES
}

\author{
EDUARD I. VOROBYOV ${ }^{1,2}$ \\ Accepted by The Astrophysical Journal
}

\begin{abstract}
Motivated by a considerable scatter in the observationally inferred lifetimes of the embedded phase of star formation, we study the duration of the Class 0 and Class I phases in upper-mass brown dwarfs and low-mass stars using numerical hydrodynamics simulations of the gravitational collapse of a large sample of cloud cores. We resolve the formation of a star/disk/envelope system and extend our numerical simulations to the late accretion phase when the envelope is nearly totally depleted of matter. We adopted a classification scheme of André et al. and calculate the lifetimes of the Class 0 and Class I phases $\left(\tau_{\mathrm{C} 0}\right.$ and $\tau_{\mathrm{CI}}$, respectively) based on the mass remaining in the envelope. When cloud cores with various rotation rates, masses, and sizes (but identical otherwise) are considered, our modeling reveals a sub-linear correlation between the Class 0 lifetimes and stellar masses in the Class 0 phase with the least-squares fit exponent $m=0.8 \pm 0.05$. The corresponding correlation between the Class I lifetimes and stellar masses in the Class I is super-linear with $m=1.2 \pm 0.05$. If a wider sample of cloud cores is considered, which includes possible variations in the initial gas temperature, cloud core truncation radii, density enhancement amplitudes, initial gas density and angular velocity profiles, and magnetic fields, then the corresponding exponents may decrease by as much as 0.3 . The duration of the Class I phase is found to be longer than that of the Class 0 phase in most models, with a mean ratio $\tau_{\mathrm{CI}} / \tau_{\mathrm{C} 0} \approx 1.5-2$. A notable exception are YSOs that form from cloud cores with large initial density enhancements, in which case $\tau_{\mathrm{C} 0}$ may be greater than $\tau_{\mathrm{CI}}$. Moreover, the upper-mass $\left(\gtrsim 1.0 M_{\odot}\right)$ cloud cores with frozen-in magnetic fields and high cloud core rotation rates may have the $\tau_{\mathrm{CI}} / \tau_{\mathrm{C} 0}$ ratios as large as 3.0-4.0. We calculate the rate of mass accretion from the envelope onto the star/disk system and provide an approximation formula that can be used in semi-analytic models of cloud core collapse.
\end{abstract}

Subject headings: circumstellar matter — planetary systems: protoplanetary disks — hydrodynamics - ISM: clouds — stars: formation

\section{INTRODUCTION}

Constraining the lifetimes associated with different phases of low-mass star formation has been traditionally one of the goals of research. In spite of much effort in this field, yet there is a considerable scatter among observationally estimated lifetimes of the embedded phase. Most previous observational studies suggested the duration of the Class 0 and Class I phases to be within the 0.1-0.7 Myr range (Wilking et al. 1989; Greene et al. 1994; Kenvon \& Hartmann 1995; Visser et al. 2002; Hatchell et al.|2007), with similar relative lifetimes (Visser et al. 2002; Hatchell et al. 2007), while some argue in favour of a significantly shorter lifetime for the Class 0 phase, $\tau_{\mathrm{C} 0}=0.01-0.06 \mathrm{Myr}$ (André \& Montmerle 1994). In the most recent work involving a large set of YSOs from different star forming clouds, Enoch et al. (2009) and Evans et al. (2009) advocated for the lifetimes of 0.1-0.2 Myr for the Class 0 sources and 0.44-0.54 Myr for the Class I ones.

The aforementioned lifetimes may vary in part due to different estimation techniques and employed wavelengths and in part due to observations being confined to different molecular clouds. The cornerstone assumption of steady star formation, adopted in most observational

\footnotetext{
${ }^{1}$ Institute for Computational Astrophysics, Saint Mary's University, Halifax, B3H 3C3, Canada; vorobyov@ap.smu.ca.

${ }^{2}$ Institute of Physics, South Federal University, Stachki 194, Rostov-on-Don, 344090, Russia.
}

studies, may break down if star formation relies heavily on external triggering, as may be the case in $\rho$ Ophiuchi (Visser et al. 2002). Furthermore, the environment and local initial conditions determine how long the Class 0 and Class I phases last.

It is therefore interesting to compare the observationally inferred lifetimes with those predicted from numerical modeling of the cloud core collapse. For instance, Masunaga \& Inutsuka (2000) performed radiation hydrodynamics simulations of the gravitational collapse of spherically symmetric cloud cores and found, based on the synthetic spectral energy distribution, that the Class 0 phase lasts around $2 \times 10^{4} \mathrm{yr}$. However, their modeling was limited to only two cloud cores. Some work in this direction was also done by Froebrich et al. (2006), who employed smooth particle hydrodynamics simulations of the fragmentation and collapse of turbulent, self-gravitating clouds and found Class 0 lifetimes to be of the order of $(2-6) \times 10^{4} \mathrm{yr}$, significantly lower than those inferred from most observations. However, they did not provide estimates for the Class I lifetime, probably due to an enormous computational cost, and their lifetimes were inferred from the mass infall rates at $\sim 300$ AU rather than from more customary diagnostics such as envelope masses or spectral properties.

In this paper, we perform a comprehensive numerical study of the duration of the embedded phase of star formation in upper-mass brown dwarfs and lowmass stars. Using numerical hydrodynamics simulations, 
we compute the gravitational collapse of a large sample of gravitationally unstable cloud cores with various initial masses, rotation rates, gas temperatures, density enhancement amplitudes, truncation radii, and strengths of frozen-in magnetic fields. The paper is organized as follows. In Sections 2 and 3 , we briefly review the numerical model and initial conditions. In Section 4, we discuss the adopted classification scheme of YSOs. In Section 5.1, we search for possible correlations between the Class 0 and Class I lifetimes, from one hand, and stellar and cloud core masses, from the other hand. The effect of varying initial conditions is considered in Section 5.2. The model envelope depletion rates are tested against several empirical functions in Section 6 and the main results are summarized in Section 7.

\section{MODEL DESCRIPTION}

Our numerical model is similar to that used recently to study the secular evolution of circumstellar disks and the mass accretion rates in $\mathrm{T}$ Tauri stars and brown dwarfs (Vorobyov \& Basu 2009a, b; Vorobyov 2009a). For the reader's convenience, we briefly summarize the basic concept and equations.

We make use of the thin-disk approximation to compute the long-term $(\sim 2 \mathrm{Myr})$ evolution of rotating, gravitationally unstable cloud cores. We start our numerical integration in the pre-stellar phase, which is characterized by a collapsing starless cloud core, continue into the embedded phase, which sees the formation of a star/disk/envelope system, and terminate our simulations in the late accretion phase, when most of the cloud core has accreted onto the star/disk system. Once the disk has self-consistently formed, it occupies the innermost regions of our numerical grid, while the infalling cloud core (the envelope) occupies the rest of the grid. This ensures that the mass accretion rate onto the disk $\dot{M}_{\text {env }}$ is self-consistently determined by the dynamics of the gas in the envelope, rather than being simply introduced as a free parameter.

We introduce a "sink cell" at $r<5$ AU and impose a free inflow inner boundary condition. As the gravitational collapse of a cloud core ensues, the matter begins to freely flows through the sink cell. We monitor the gas surface density in the sink cell and when its value exceeds a critical value $\Sigma_{\text {cr }}$ for the transition from isothermal to adiabatic evolution (see Equation [5]), we introduce a central star. In the subsequent evolution, ninety per cent of the gas that crosses the inner boundary is assumed to land onto the central star plus the inner axisymmetric disk at $r<5 \mathrm{AU}$. The mass ratio of the inner axisymmetric disk relative to the star is a few per cent, so that most of the accreted mass is accumulated by the star. The inner disk is dynamically inactive; it contributes only to the total gravitational potential and its use is necessary to ensure a smooth behaviour of the gravity force down to the stellar surface. The other $10 \%$ of the accreted gas is assumed to be carried away with protostellar jets. The latter are triggered only after the formation of the central star.

The basic equations of mass and momentum transport in the thin-disk approximation are

$$
\frac{\partial \Sigma}{\partial t}=-\nabla_{p} \cdot\left(\Sigma \boldsymbol{v}_{p}\right)
$$

$$
\Sigma \frac{d \boldsymbol{v}_{p}}{d t}=-\nabla_{p} \mathcal{P}+\Sigma \boldsymbol{g}_{p}-\frac{Z}{4 \pi} \nabla_{p} B_{z}^{2}+\frac{B_{z} \boldsymbol{B}_{p}}{2 \pi}+(\nabla \cdot \boldsymbol{\Pi})(2,)
$$

where $\Sigma$ is the mass surface density, $\mathcal{P}=\int_{-Z}^{Z} P d z$ is the vertically integrated form of the gas pressure $P$, $Z$ is the radially and azimuthally varying vertical scale height, $\boldsymbol{v}_{p}=v_{r} \hat{\boldsymbol{r}}+v_{\phi} \hat{\boldsymbol{\phi}}$ is the velocity in the disk plane, $\boldsymbol{g}_{p}=g_{r} \hat{\boldsymbol{r}}+g_{\phi} \hat{\boldsymbol{\phi}}$ is the gravitational acceleration in the disk plane, and $\nabla_{p}=\hat{\boldsymbol{r}} \partial / \partial r+\hat{\boldsymbol{\phi}} r^{-1} \partial / \partial \phi$ is the gradient along the planar coordinates of the disk. The gravitational acceleration $\boldsymbol{g}_{p}$ includes the gravity of a central point object (when formed), the gravity of the inner inactive disk $(r<5 \mathrm{AU})$, and the self-gravity of a circumstellar disk and envelope. The latter component is found by solving the Poisson integral using the convolution theorem. The disk is pierced with a magnetic field, which has only the vertical component $B_{z}$ in the disk and both the vertical and planar $\left(\boldsymbol{B}_{p}=B_{r} \hat{\boldsymbol{r}}+B_{\phi} \hat{\boldsymbol{\phi}}\right)$ components at the top and bottom surfaces of the disk. Because of our assumption of flux freezing and a spatially uniform flux-to-mass ratio $\alpha_{\mathrm{m}}$, the vertical and planar magnetic field components are easily determined from the relations $B_{z}=\alpha_{\mathrm{m}} 2 \pi G^{1 / 2} \Sigma$ and $\boldsymbol{B}_{p}=-\alpha_{\mathrm{m}} \boldsymbol{g}_{p} / G^{1 / 2}$, respectively (see Vorobyov \& Basu 2006, for details).

The viscous stress tensor $\boldsymbol{\Pi}$ is expressed as

$$
\boldsymbol{\Pi}=2 \Sigma \nu\left(\nabla v-\frac{1}{3}(\nabla \cdot v) \mathbf{e}\right),
$$

where $\nabla v$ is a symmetrized velocity gradient tensor, $\mathbf{e}$ is the unit tensor, and $\nu$ is the kinematic viscosity. The components of $(\nabla \cdot \boldsymbol{\Pi})_{p}$ in polar coordinates $(r, \phi)$ can be found in Vorobyov \& Basu (2009a). We make no specific assumptions about the source of viscosity and parameterize the magnitude of kinematic viscosity using a usual form of the $\alpha$-prescription

$$
\nu=\alpha c_{\mathrm{s}, \mathrm{eff}} Z,
$$

where $c_{\mathrm{s} \text {,eff }}^{2}=\gamma \mathcal{P} / \Sigma$ is the square of the effective sound speed and the ratio of specific heats is set to $\gamma=1.4$. We note that $c_{\mathrm{s} \text {,eff }}$ in this formula is a dynamically varying quantity and is calculated at every time step during the evolution.

In this paper, we use a spatially and temporally uniform $\alpha=0.005$. This choice is based on the recent work of Vorobyov \& Basu (2009a), who studied numerically the secular evolution of viscous and self-gravitating disks. They found that if circumstellar disks around solar-mass protostars could generate and sustain turbulence then the temporally and spatially averaged $\alpha$ should lie in the $10^{-3}-10^{-2}$ limits. Smaller values of $\alpha\left(\lesssim 10^{-4}\right)$ have little effect on the resultant mass accretion history, while larger values $\left(\alpha \gtrsim 10^{-1}\right)$ destroy circumstellar disks during less than 1.0 Myr of evolution and are thus inconsistent with mean disk lifetimes of the order of 2-3 Myr.

Equations (11) and (2) are closed with a barotropic equation that makes a smooth transition from isothermal to adiabatic evolution at $\Sigma=\Sigma_{\mathrm{cr}}$ :

$$
\mathcal{P}=c_{\mathrm{s}}^{2} \Sigma+c_{\mathrm{s}}^{2} \Sigma_{\mathrm{cr}}\left(\frac{\Sigma}{\Sigma_{\mathrm{cr}}}\right)^{\gamma},
$$

We note that in this equation (and further in the text) $c_{\mathrm{s}}^{2}=R T_{0} / \mu$ is the sound speed at the beginning of numerical simulations, which depends on the adopted initial 
gas temperature $T_{0}$ of a cloud core and the mean molecular weight $\mu$. The value of $\Sigma_{\mathrm{cr}}$ is calculated during the numerical simulations as $\Sigma_{\mathrm{cr}}=m_{\mathrm{H}} \mu n_{\mathrm{cr}} 2 Z$, where the critical volume number density $n_{\mathrm{cr}}$ is set to $10^{11} \mathrm{~cm}^{-3}$ (Larson 2003) and the mean molecular weight is set to 2.33. The scale height $Z$ is calculated using the assumption of vertical hydrostatic equilibrium (see Vorobyov 2009a). We note that $Z$ is an increasing function of radius, which makes $\Sigma_{\text {cr }}$ to increase with radius as well. In practice, this means that the inner disk regions are significantly warmer than the outer regions, since the optically thick regime in the inner regions is achieved at lower $\Sigma$. This in turn impedes the development of gravitational instability and fragmentation in the inner disk, in agreement with more sophisticated numerical simulations and theoretical predictions that directly solve for the energy balance equation (see e.g. Boley et al. 2009).

Equations (11) and (2) are solved in polar coordinates $(r, \phi)$ on a numerical grid with $256 \times 256$ grid zones. We use the method of finite differences with a time-explicit solution procedure similar in methodology to the ZEUS code, with the advection and the force terms treated separately using the operator-split method. Advection is performed using the second-order van Leer scheme. A small amount of artificial viscosity that spreads shocks over two grid zones is added. However, the resulted artificial viscosity torques are negligible in comparison to gravitational and $\alpha$-viscosity ones. The radial points are logarithmically spaced. The innermost grid point is located at $r_{\mathrm{sc}}=5 \mathrm{AU}$, and the size of the first adjacent cell varies in the $0.12-0.17 \mathrm{AU}$ range depending on the cloud core size.

\section{INITIAL CONDITIONS}

We start our numerical simulations from starless, gravitationally bound cloud cores, which have surface densities $\Sigma$ and angular velocities $\Omega$ typical for a collapsing, axisymmetric, magnetically supercritical core (Basu 1997)

$$
\begin{gathered}
\Sigma=\frac{r_{0} \Sigma_{0}}{\sqrt{r^{2}+r_{0}^{2}}}, \\
\Omega=2 \Omega_{0}\left(\frac{r_{0}}{r}\right)^{2}\left[\sqrt{1+\left(\frac{r}{r_{0}}\right)^{2}}-1\right],
\end{gathered}
$$

where $\Omega_{0}$ is the central angular velocity, $r_{0}$ is the radius of central near-constant-density plateau defined as $r_{0}=\sqrt{A} c_{\mathrm{s}}^{2} /\left(\pi G \Sigma_{0}\right)$. We note that when $r \gg r_{0}$, the resultant gas surface density becomes $\Sigma=\sqrt{A} c_{\mathrm{s}}^{2} /(\pi G r)$. This profile can be derived from the following gas volume density distribution $\rho=A c_{\mathrm{s}}^{2} /\left(2 \pi G r^{2}\right)$, if the latter is integrated in the vertical direction assuming a local vertical hydrostatic equilibrium, i.e. $\rho=\Sigma /(2 Z)$ and $Z=c_{\mathrm{s}}^{2} /(\pi G \Sigma)$. Therefore, our initial density configuration is characterized by a factor of $A$ positive density enhancement as compared to that of the singular isothermal sphere $\rho_{\text {SIS }}=c_{\mathrm{s}}^{2} /\left(2 \pi G r^{2}\right)($ Shu 1977) $)$.

Cloud core are characterized by the ratio of rotational to gravitational energy $\beta=E_{\text {rot }} /\left|E_{\text {grav }}\right|$, where the rota- tional and gravitational energies are calculated as

$$
E_{\mathrm{rot}}=2 \pi \int_{r_{\mathrm{sc}}}^{r_{\mathrm{out}}} r a_{\mathrm{c}} \Sigma r d r, \quad E_{\mathrm{grav}}=-2 \pi \int_{r_{\mathrm{sc}}}^{\mathrm{r}_{\mathrm{out}}} r g_{r} \Sigma r d r .
$$

Here, $a_{\mathrm{c}}=\Omega^{2} r$ is the centrifugal acceleration, and $r_{\text {out }}$ is the outer cloud core radius. Cloud cores are initially isothermal, with the gas temperature varying between $T_{0}=10 \mathrm{~K}$ and $18 \mathrm{~K}$, depending on the model.

We present results from 10 sets of models, the parameters of which are summarized in Table 1 . Every model set has a distinct ratio $\beta$, the adopted values of which lie within the limits inferred by Caselli et al. (2002) for dense molecular cloud cores, $\beta=\left(10^{-4}-0.07\right)$. We note that by construction $\beta=0.91 \Omega_{0}^{2} r_{0}^{2} / c_{\mathrm{s}}^{2}$ Vorobyov $2009 \mathrm{~b})$. In addition, each set of models is characterized by a distinct ratio $r_{\text {out }} / r_{0}$ in order to generate gravitationally unstable truncated cores of similar form. For most model sets, we choose $r_{\text {out }} / r_{0}=6.0$.

Every model set contains several individual models ${ }^{3}$ that are characterized by distinct masses $M_{\mathrm{cl}}$, outer radii $r_{\text {out }}$, and central angular velocities $\Omega_{0}$, but have equal $\beta$ and $r_{\text {out }} / r_{0}$. The actual procedure for generating a specific cloud core with a given value of $\beta$ is as follows. First, we choose the outer cloud core radius $r_{\text {out }}$ and find $r_{0}$ from the condition $r_{\text {out }} / r_{0}=$ const. Then, we find the central surface density $\Sigma_{0}$ from the relation $r_{0}=$ $\sqrt{A} c_{\mathrm{s}}^{2} /\left(\pi G \Sigma_{0}\right)$ and determine the resulting cloud core mass $M_{\mathrm{cl}}$ from Equation (6). Finally, the central angular velocity $\Omega_{0}$ is found from the condition $\beta=0.91 \Omega_{0}^{2} r_{0}^{2} / c_{\mathrm{s}}^{2}$.

In total, we have simulated numerically the time evolution of 106 cloud cores spanning a range of initial masses between $0.06 M_{\odot}$ and $3.9 M_{\odot}$. We have split this mass interval into 14 bins of equal size $d M_{\mathrm{cl}}$. The resulting initial cloud core mass function $d N / d M_{\mathrm{cl}}=M_{\mathrm{cl}}^{-m}$ is shown in Figure 1 by filled circles. The dashed lines present the least-squares best fits to low-mass $\left(0.06 M_{\odot}<M_{\mathrm{cl}} \leq\right.$ $0.6 M_{\odot}$, left line) cloud cores and intermediate- and upper-mass $\left(0.6 M_{\odot}<M_{\mathrm{cl}}<3.9 M_{\odot}\right.$, right line) cloud cores. The corresponding exponents are $m=0.5 \pm 0.1$ and $m=1.6 \pm 0.2$, respectively. Our model cloud core function is similar to that inferred from nearby starforming regions (e.g. Enoch et al. 2006). The masses of our model cloud cores have been selected from a mass distribution that mimics the observed core mass distribution in order to perform averages of the model parameters (such as disk masses, Class 0/I lifetimes, etc.) weighted with the cloud core mass function.

\section{CLASSIFICATION SCHEMES}

Modern classification schemes of young stellar objects are designed to distinguish between the four main physical phases of their evolution: the cloud core collapse and formation of a protostar and a disk (Class 0 and Class I), envelope clearing and disk accretion onto the star (Class II), and disk dissipation and planet formation (Class III). The spectral energy distribution is often used to relate a YSOs to a particular class (see Evans et al. 2009, for a thorough review). For instance, Lada (1987) used the spectral index $\mathrm{n}$ (defined by $\nu F_{\nu} \propto \nu^{n}$, where $F_{\nu}$ was

3 The exact number of these models is specified in the last column of Table 1 
TABLE 1

MODEL PARAMETERS

\begin{tabular}{cccccccccc}
\hline \hline Set & $\beta$ & $\Omega_{0}$ & $r_{0}$ & $M_{\mathrm{cl}}$ & $r_{\text {out }} / r_{0}$ & $T_{0}$ & $A$ & $\alpha_{m}$ & $\mathrm{~N}$ \\
\hline 1 & $2.17 \times 10^{-3}$ & $0.57-3.30$ & $583-3360$ & $0.34-1.9$ & 6 & 10 & 2 & 0 & 8 \\
2 & $3.20 \times 10^{-3}$ & $0.57-5.60$ & $411-4110$ & $0.24-2.4$ & 6 & 10 & 2 & 0 & 12 \\
$2 \mathrm{~T}$ & $3.20 \times 10^{-3}$ & $1.30-10.5$ & $411-3360$ & $0.43-3.5$ & 6 & 18 & 2 & 0 & 11 \\
$2 \mathrm{~A}$ & $3.20 \times 10^{-3}$ & $0.98-9.6$ & $342-3360$ & $0.40-3.9$ & 6 & 10 & 8 & 0 & 12 \\
$2 \mathrm{MF}$ & $3.20 \times 10^{-3}$ & $0.57-5.7$ & $411-4110$ & $0.24-2.4$ & 6 & 10 & 2 & 0.3 & 12 \\
$2 \mathrm{E}$ & $3.20 \times 10^{-3}$ & $1.0-5.7$ & $411-2230$ & $0.5-2.8$ & 12 & 10 & 2 & 0 & 9 \\
3 & $6.90 \times 10^{-3}$ & $1.11-16.6$ & $206-3086$ & $0.12-1.8$ & 6 & 10 & 2 & 0 & 12 \\
4 & $9.90 \times 10^{-3}$ & $1.10-25.0$ & $164-3771$ & $0.095-2.2$ & 6 & 10 & 2 & 0 & 10 \\
5 & $1.34 \times 10^{-2}$ & $1.33-35.0$ & $137-3600$ & $0.079-2.1$ & 6 & 10 & 2 & 0 & 10 \\
6 & $2.22 \times 10^{-2}$ & $1.56-45.0$ & $102-3940$ & $0.06-2.3$ & 6 & 10 & 2 & 0 & 10 \\
\hline
\end{tabular}

Note. - All distances are in AU, angular velocities in $\mathrm{km} \mathrm{s}^{-1} \mathrm{pc}^{-1}$, masses in $M_{\odot}$, initial gas temperatures $T_{0}$ in Kelvin and $N$ is the (n) All in

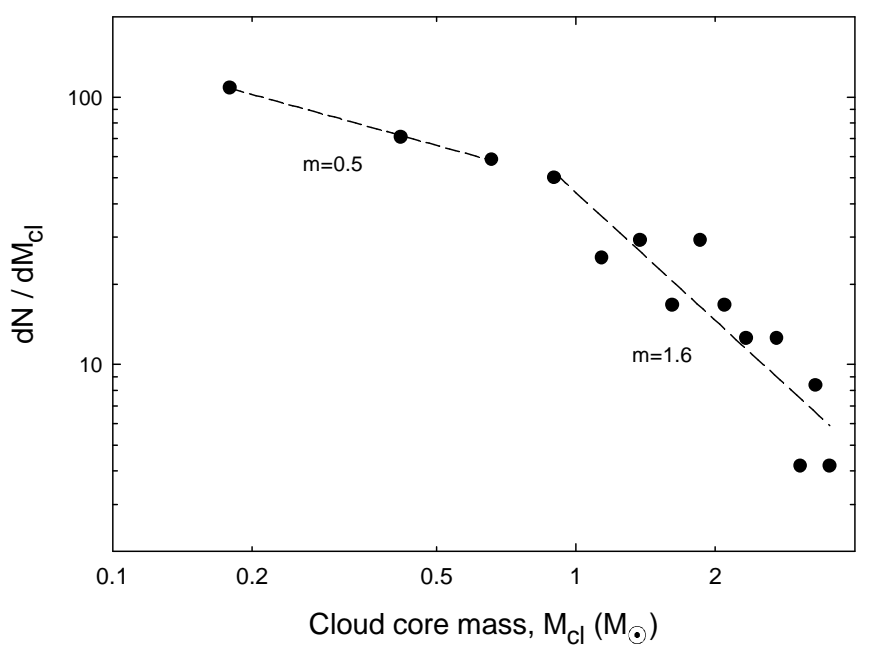

FIG. 1.- Initial cloud core mass function $d N / d M_{\mathrm{cl}}=M_{\mathrm{cl}}^{-m}$ for 106 model cloud cores organized in 14 bins of equal size $d M_{\mathrm{cl}}^{\mathrm{cl}}$. The parameters of the cloud cores are listed in Table 1 The dashed lines present the least-squares best fits to low-mass cloud cores $\left(0.06 M_{\odot}<M_{\mathrm{cl}} \leq 0.6 M_{\odot}\right.$, left line) and intermediate- and uppermass cloud cores $\left(0.6 M_{\odot}<M_{\mathrm{cl}}<3.9 M_{\odot}\right.$, right line $)$.

the infrared flux density at frequency $\nu$ ) to distinguish between Class I, Class II, and Class III objects. Later, André et al. (1993) introduced the ratio of submillimeter to bolometric luminosity $L_{\text {submm }} / L_{\text {bol }}$ to split the early embedded stage into the Class 0 and Class I phases based on the mass reservoir remaining in the envelope. This separation was originally motivated by the discovery of very young protostars in collapsing cloud cores that were previously being thought of as starless. But it also makes physical sense because the Class 0 phase can be identified with a period of temporally increasing $L_{\mathrm{bol}}$ and Class I phase with a later period of decreasing $L_{\mathrm{bol}}$ (Vorobyov \& Basu 2005). Finally, Myers \& Ladd (1993) suggested the use of a bolometric temperature $T_{\text {bol }}$, defined as the temperature of a blackbody with the same flux-weighted mean frequency as the actual spectral energy distribution.

In our case, it is difficult to use any of the aforementioned schemes due to a simplified treatment of the thermal physics in our numerical simulations. Moreover, the large inclination dependence introduced by the outflow cavities prevents both $T_{\text {bol }}$ and $L_{\text {bol }} / L_{\text {submm }}$ from being good evolutionary indicators (Dunham et al. 2010). Therefore, we use the classification breakdown suggested also by André et al. (1993) and based on the mass re-

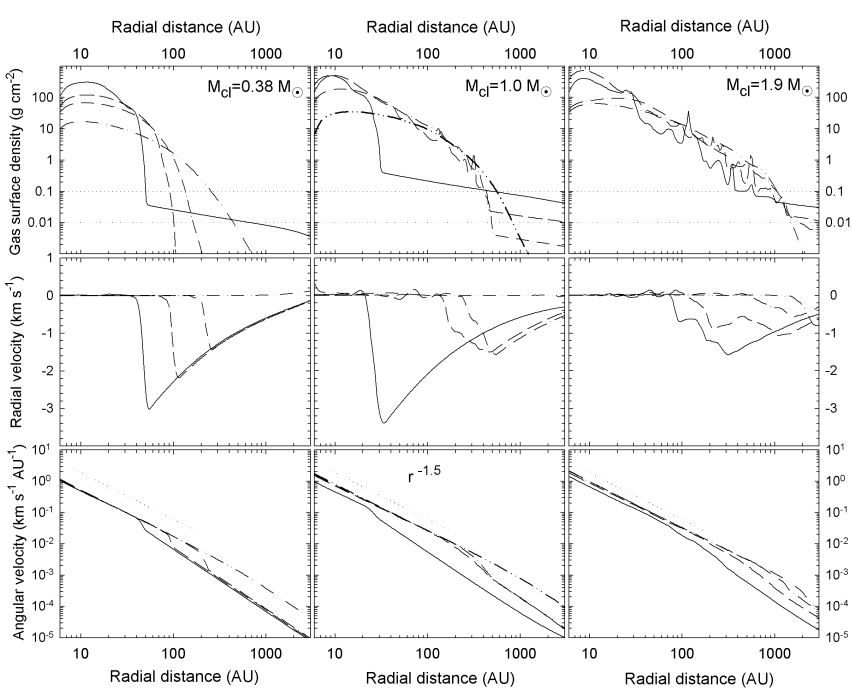

FIG. 2.- Azimuthally averaged profiles of the gas surface density (top row), radial velocity (middle row), and azimuthal velocity (bottom row) for three models from model set 2. The initial cloud core masses are indicated in the top row. Four different evolution times (since the formation of the protostar) are shown with solid (0.1 Myr), dashed (0.2 Myr), dash-dotted (0.3 Myr), and dashdot-dotted (1.0 Myr) lines. The dotted horizontal lines in the top row mark the two adopted values of the critical gas surface density $\Sigma_{\mathrm{d} 2 \mathrm{e}}$ for the disk to envelope transition. The dotted line in the bottom row presents the angular velocity in the gravitational field of a $4.0 M_{\odot}$ star.

maining in the envelope

$$
\begin{array}{ll}
\text { Class 0 } & M_{\mathrm{env}} \geq 0.5 M_{\mathrm{cl}} \\
\text { Class I } & 0.1 M_{\mathrm{cl}} \leq M_{\mathrm{env}}<0.5 M_{\mathrm{cl}} \\
\text { Class II } & M_{\mathrm{env}}<0.1 M_{\mathrm{cl}} .
\end{array}
$$

According to this scheme (hereafter, AWTB scheme), transition between Class 0 and Class I objects occurs when the envelope mass $M_{\text {env }}$ decreases to half of the initial cloud core mass $M_{\mathrm{cl}}$. The Class II phase ensues by the time when the infalling envelope nearly clears and its total mass drops below $10 \%$ of the initial cloud core mass. Of course, we acknowledge that these numbers are somewhat arbitrary and the use of other classification diagnostics may introduce a systematic shift to our derived lifetimes. e spectral energy distribution.

In order to use the AWTB scheme, we need to know which part of our numerical grid is occupied by the disk and which part belongs to the infalling envelope at any time instance of the evolution (note that we know accurately the stellar mass). This turned out to be a nontrivial task. An intuitive step of calculating the rota- 
tion profile and separating between Keplerian and subKeplerian regions of the grid sometimes failed to work due to large radial motions occasionally present in the disk. Besides, the disk is not exactly Keplerian due to a noticeable contribution from its self-gravity. Therefore, we have developed a hybrid method that makes use of the radial velocity field in the envelope and a critical surface density for the disk-to-envelope transition.

Figure 2 illustrates our idea and shows the azimuthallyaveraged gas surface density $\bar{\Sigma}$ (top row), radial velocity $\bar{v}_{r}$ (middle row), and azimuthal velocity $\bar{v}_{\phi}$ (bottom row) distributions as a function of radial distance $r$ for three representative models from model set 2 . The initial cloud core masses $M_{\mathrm{cl}}$ are shown in the top row to distinguish between the models. For each model, the radial profiles at four different evolutionary times (elapsed since the protostar formation) are plotted with the solid (0.1 Myr), dashed (0.2 Myr), dash-dotted (0.3 Myr), and dash-dot-dotted (1.0 Myr) lines. From the radial distribution of $\bar{\Sigma}$ it is seen that the disk is compact and dense upon formation but later it spreads radially outward due to the action of viscous torques, which are positive in the outer disk regions (Vorobyov \& Basu 2009a). The disk has a near-Keplerian rotation profile as can be seen from the comparison of $\bar{v}_{\phi}$ with the dotted line, the latter showing the angular velocity in the gravitational field of a $4.0 M_{\odot}$ star. It is worth noting that the evolution of the unmagnetized models seems to approach a self-similar behavior in the infalling envelope. This is evident in the $M=1.0 M_{\odot}$ model, which radial velocity and surface density distributions approach a free-fall profile proportional to $r^{-1 / 2}$.

In our numerical simulations, the disk occupies the inner regions (small $r$ ), while the envelope - the outer region of the computational grid (large $r$ ). A large drop in $\bar{v}_{\mathrm{r}}$ (by absolute value) correspond to the radial position where the infalling envelope lands onto the disk (the socalled accretion shock). The shock position propagates radially outward, reflecting the disk expansion. It is also evident that the shock front becomes less sharp with increasing cloud core mass, reflecting an increasingly complex spiral structure of massive disks (recall that Figure2 presents azimuthally averaged profiles). It is seen that, in general, the envelope is characterized by large negative $\bar{v}_{r}$ due to gravitationally driven collapse and low $\bar{\Sigma}$ due to gas depletion. On the other hand, the disk has much smaller $\bar{v}_{r}$ due to near-(but never complete)centrifugal balance and larger $\bar{\Sigma}$ due to mass accumulation. The outer parts of the disk expand and the overall disk density decreases with time due to ongoing angular momentum redistribution. In general, we find that massive disks are not in steady state but exhibit substantial radial pulsations due to the time-dependent strength of gravitational instability in the disk. The gas surface density profile declines with radius but some nonmonotonic behavior is evident for upper-mass models due to fragmentation in the outer parts of the disk.

Having analyzed these data, we adopt the following procedure to distinguish between the infalling envelope and the disk. We march from the outer computational boundary toward the inner one (in the direction of decreasing $r$ ) and determine the radial distance at which the following two criteria are fulfilled simultaneously:

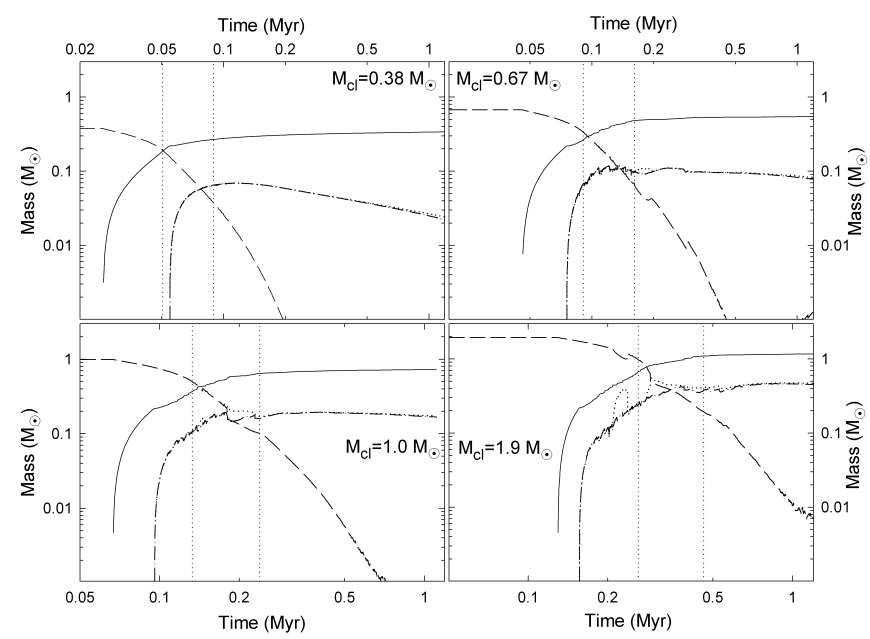

FIG. 3.- Time evolution of the stellar mass (solid lines), envelope mass (dashed lines) and disk mass (dotted and dash-dotted lines) for four models from model set 2. The initial cloud core masses $M_{\mathrm{cl}}$ are indicated in each panel. Two disk masses based on the value of the critical gas surface density $\Sigma_{\mathrm{d} 2 \mathrm{e}}$ are shown by the dash-dotted lines $\left(\Sigma_{\mathrm{d} 2 \mathrm{e}}=0.1 \mathrm{~g} \mathrm{~cm}^{-2}\right)$ and dotted lines $\Sigma_{\mathrm{d} 2 \mathrm{e}}=0.01 \mathrm{~g} \mathrm{~cm}^{-2}$. The vertical dotted lines mark the onset of the Class I phase (left line) and Class II phase (right line).

$\bar{\Sigma}>\Sigma_{\mathrm{d} 2 \mathrm{e}}$ and $\bar{v}_{r}>0$. This radial distance is set to represent the disk's tentative outer radius $r_{\mathrm{d}}$ (at a given time instance of the evolution). For the critical transitional density $\Sigma_{\mathrm{d} 2 \mathrm{e}}$, we choose $0.01 \mathrm{~g} \mathrm{~cm}^{-2}$ or $0.1 \mathrm{~g} \mathrm{~cm}^{-2}$ shown in Figure 2 with horizontal dotted lines. All material that is located within $r \leq r_{\mathrm{d}}$ is considered to belong to the disk. This procedure is repeated each time we want to determine the disk and envelope masses and it has proven to be most accurate in our circumstances.

Figure 3 presents the integrated disk (dotted and dashdotted lines), envelope (dashed lines), and stellar masses (solid lines) as a function of time elapsed since the beginning of numerical simulations in four representative models from model set 2 . The initial cloud core masses $M_{\mathrm{cl}}$ are indicated in each panel. Two different disk masses are shown based on the critical density $\Sigma_{\mathrm{d} 2 \mathrm{e}}=0.01 \mathrm{~g} \mathrm{~cm}^{-2}$ (dotted lines) and $\Sigma_{\mathrm{d} 2 \mathrm{e}}=0.1 \mathrm{~g} \mathrm{~cm}^{-2}$ (dash-dotted lines). The vertical dotted lines mark the onset of the Class I phase (left line) and Class II phase (right line). It is seen that the appearance of the star occurs at progressively later evolution times with increasing cloud core mass. This is due to the fact that the free-fall time $\tau_{\mathrm{ff}}$ of a cloud core is linearly proportional to its mass (see the Appendix). Indeed, in the $M_{\mathrm{cl}}=0.38 M_{\odot}$ model, the star appears at $t=0.026 \mathrm{Myr}$, whereas in the $M_{\mathrm{cl}}=1.9 M_{\odot}$ model the star appears at $t=0.129 \mathrm{Myr}$, which is in good agreement with what is expected from the linear proportionality between $\tau_{\mathrm{ff}}$ and $M_{\mathrm{cl}}$. It is also seen that the difference between disk masses based on the two values of $\Sigma_{\mathrm{d} 2 \mathrm{e}}$ is mostly indistinguishable, apart from several episodes when some noticeable mismatch is evident. These episodes occur only in the early evolution (and in massive disks) and are associated with a transitory disk expansion after the accretion of massive fragments (formed due to disk fragmentation) by the central protostar (Vorobyov \& Basu 2006).

Figure 3 also shows that the disk mass is always lower than that of the protostar. This is typical of self-gravitating disks in which self-gravity is computed self-consistently (Vorobyov 2009a; Kratter et al. 2009), 
rather than being calculated using an effective viscosity proportional to the Toomre $Q$-parameter as in Lin \& Pringle (1990). In the latter case, the stellar mass may be substantially underestimated in models with large disk-to-star mass ratios, whereas the disk mass agrees better with that obtained from self-consistent calculations of disk self-gravity (Vorobyov 2009d). A net result of this discrepancy is that the disk mass starts to considerably exceed that of the star soon after its formation, which contradicts observations. We note that the time difference between the star and disk formation events is somewhat overestimated in our numerical simulations due to the use of the sink cell. The rate at which the envelope mass declines with time is clearly dependent on $M_{\mathrm{cl}}$ - the envelope depletes much slower in models with greater $M_{\mathrm{cl}}$.

\section{LIFETIMES OF THE EMBEDDED PHASE}

In this section, we calculate lifetimes of the Class 0 and Class I phases based on the AWTB criterion (see Equation 9]). Depending on the adopted value of the critical density $\Sigma_{\mathrm{d} 2 \mathrm{e}}$ for the disk-to-envelope transition (0.01 $\mathrm{g} \mathrm{cm}^{-2}$ or $\left.0.1 \mathrm{~g} \mathrm{~cm}^{-2}\right)$, the resulting lifetimes may differ by a factor of unity. In order to minimize the uncertainty, we use the arithmetically averaged values. For model set 6 , however, we use lifetimes based on $\Sigma_{\mathrm{d} 2 \mathrm{e}}=0.01 \mathrm{~g} \mathrm{~cm}^{-2}$. This is because model set 6 produces most massive and extended disks (due to considerable rotation rates of the corresponding cloud cores), which are best described by the smaller value of $\Sigma_{\mathrm{d} 2 \mathrm{e}}$.

For the adopted initial (isothermal) gas surface density distribution of the form $\Sigma \propto r^{-1}$, one may expect that Class 0 and Class I lifetimes $\left(\tau_{\mathrm{C} 0}\right.$ and $\tau_{\mathrm{CI}}$, respectively) scale linearly with cloud core mass $M_{\mathrm{cl}}$. Indeed, for this case, the enclosed mass is proportional to radius and therefore the crossing-time $t_{\mathrm{cr}}=r / c_{s}$ of an infalling gas shell is roughly proportional to the enclosed mass. A more rigorous derivation given in the Appendix shows that the free-fall time $\tau_{\mathrm{ff}}$ is linearly proportional to $M_{\mathrm{cl}}$, inverse proportional to the initial density enhancement amplitude $A$, and scales with the initial gas temperature as $T^{-3 / 2}$. If the ratios $\tau_{\mathrm{C} 0} / \tau_{\mathrm{ff}}$ and $\tau_{\mathrm{CI}} / \tau_{\mathrm{ff}}$ are independent of cloud core mass (which is not always the case, see Figure (7), then a linear relation between lifetimes and cloud core masses may also be anticipated.

However, the Class 0/I lifetimes are often inferred from observations using stellar characteristics, and not those of cloud cores. Therefore, it is desirable to relate lifetimes with observationally meaningful stellar properties such as stellar masses. The existence of a linear dependence between lifetimes and stellar masses, similar to that anticipated for cloud core masses, is less obvious $a$ priori. First, the stellar mass varies significantly during the Class 0 phase and, to a lesser extent, during the Class I phase (see Figure 3). It could be observationally difficult to know the exact evolutionary instance of the forming star, i.e., whether the star is near the beginning of, say, the Class 0 phase or in the midway of it. Second, not all infalling mass goes into the star. A significant fraction lands onto the disk, and the disk-tostar mass ratio also varies significantly during the early evolution (Figure 3, see also Vorobyov (2009a)). These facts suggest that a linear correlation between lifetimes and stellar masses can be theoretically anticipated only if the latter are the final masses. On the other hand, using the final stellar masses in the lifetime-stellar mass dependence may be misleading, since observations would provide a whole spectrum of masses at various evolution instances of the Class 0 and Class I phases, and not only at the end of them.

To complicate things even further, the lifetime-stellar mass correlation may be influenced by other cloud core parameters such as magnetic fields and the initial rate of rotation, the effects of which are difficult to evaluate theoretically. And finally, although the adopted $\Sigma \propto r^{-1}$ scaling of the initial gas surface density with radius is borne out observationally (Bacmann et al. 2000; Dapp \& Basu 2009) and theoretically (Basu 1997), there is no guarantee that other scaling laws could not be realized in nature. This justifies a detailed numerical investigation into to the problem.

\subsection{Lifetimes versus stellar masses}

In this section, we present numerically derived relations between Class 0 and Class I lifetimes and corresponding stellar masses, $M_{*, \mathrm{C} 0}$ and $M_{*, \mathrm{CI}}$. Since stellar masses quickly increase with time (see Figure 3), we choose to consider five typical stellar masses based on the ratio $M_{\mathrm{env}} / M_{\mathrm{cl}}$. This ratio is useful because it gradually decreases with time and can be used as a tracer of the early evolution of a protostar. In particular, we consider three stellar masses derived when the ratio $M_{\mathrm{env}} / M_{\mathrm{cl}}$ becomes equal $0.9,0.7$, and 0.5 . These stellar masses are meant to represent typical ones in the beginning, in the midway, and at the end of the Class 0 phase, respectively. For the typical stellar masses in the Class I phase, we choose three values based on $M_{\mathrm{env}} / M_{\mathrm{cl}}=0.5$, 0.3 , and 0.1 . We note that we use the same stellar masses at the end of the Class 0 phase and at the beginning of the Class I phase, but the corresponding lifetimes are different. Of course, these five stellar masses cannot represent the whole possible spectrum of stellar masses that can be observationally detected in the embedded phase of star formation. Nevertheless, they can help to reduce a possible bias towards a particular evolution stage and can help to make our lifetime-stellar mass relation more observationally meaningful.

Figure 4 presents our model lifetimes (in Myr) of the Class 0 phase (top panels) and Class I phase (bottom panels) versus stellar masses (in $M_{\odot}$ ) for six model sets. More specifically, model set 1 is plotted by red trianglesup, model set 2-by blue circles, model set 3-by green squares, model set 4-by plus signs, model set 5-by pink diamonds, and model set 6 - by brown trianglesdown. Stellar masses in each panel are derived at specific evolutionary times as specified by the ratio of envelope mass to cloud core mass $M_{\text {env }} / M_{\mathrm{cl}}$. In particular, the upper-left panel employs stellar masses obtained at the beginning of the Class 0 phase, respectively. The uppermiddle and upper-right panels use stellar masses derived in the midway and at the end of the Class 0 phase. The same age segregation is used in the bottom row of panels only now the stellar masses are related to the Class I phase.

Each symbol of same color and shape within a given set of models represents an individual object, which has formed from a cloud core of distinct mass, rotation rate, and outer radius. 


\section{Stellar mass $\left(\mathrm{M}_{\odot}\right)$}

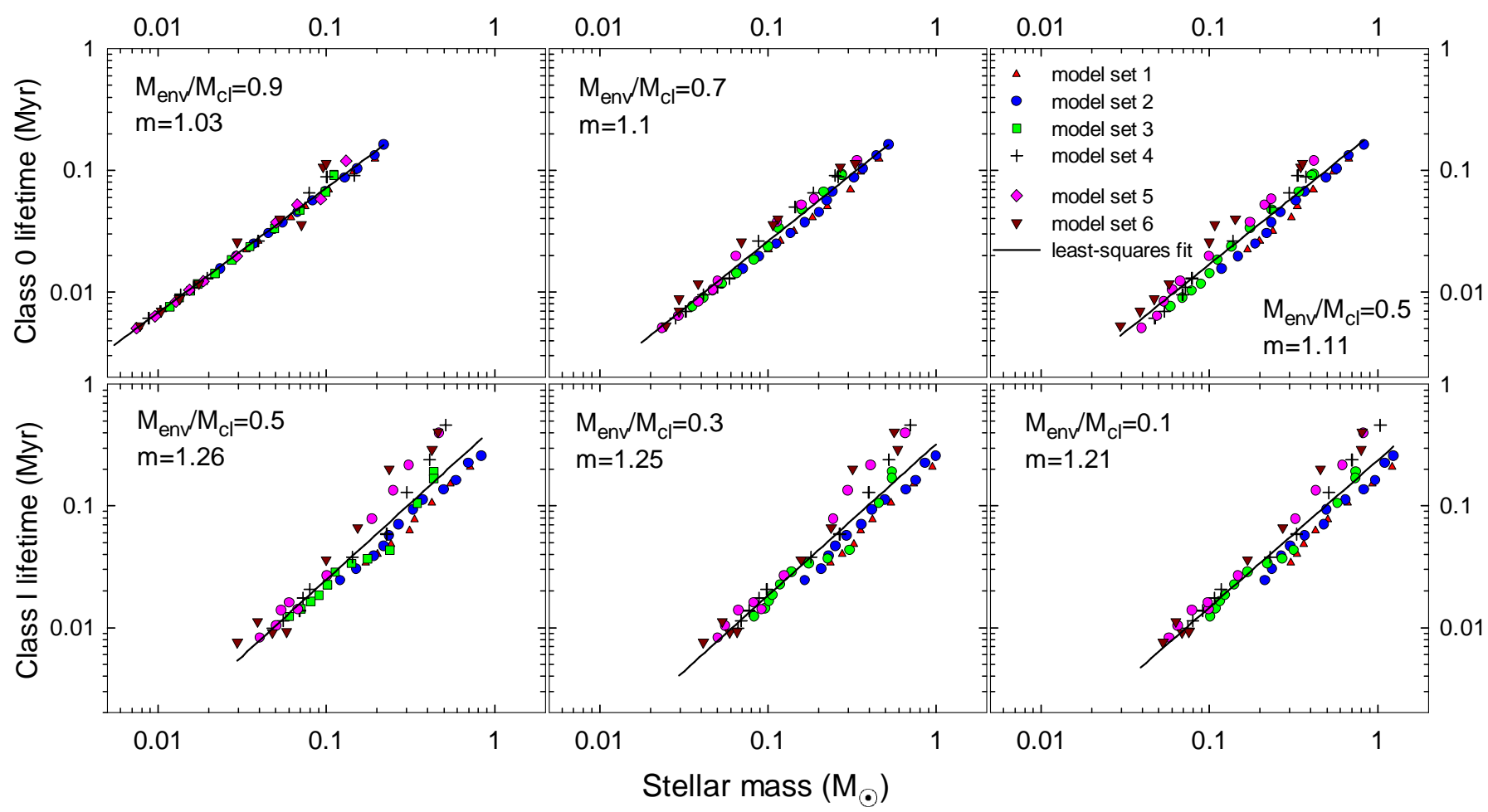

Fig. 4. - Lifetimes of the Class 0 phase (top row) and Class I phase (bottom row) versus stellar masses calculated at the beginning (right column), in the midway (middle column), and at the end (right column) of each evolution phase. The data for six model sets (see Table 1 ) are shown. The solid lines present the least-squares fits to these data. The ratio $M_{\mathrm{env}} / M_{\mathrm{cl}}$ of the envelope mass to the initial cloud core mass and the exponent $m$ of the least-squares fit are indicated in every panel.

There are two interesting features in Figure 4 that deserve particular attention. First, and most important one, is a notable dependence of the derived lifetimes on the mass of the central object. The least-squares best fits (solid lines) yield a near-linear correlations between the Class 0 lifetimes and stellar masses and a somewhat steeper correlation for the case of the Class I lifetimes. The corresponding exponents $m$ are indicated in every panel. Second interesting feature in Figure 4 is seen along the line of constant $M_{*}$ - there is a trend for models with greater ratio of rotational to gravitational energy $\beta$ to have greater Class 0/I lifetimes. This effect is especially prominent for the Class I objects and is not unexpected. Cloud cores that have more energy stored in rotation are expected to retain the envelope for a longer time due to an increased centrifugal force, thus prolonging the embedded phase of star formation.

The correlations shown in each panel of Figure 4 may not be observationally meaningful when taken separately, because they are derived for stars of specific age. As noted above, observations would not be biased towards any specific instance of protostellar evolution but are likely to detect a whole spectrum of stellar ages. To make our predictions more observationally significant, we plot in Figure 5 the Class 0 (top) and Class I (bottom) lifetimes versus all stellar masses, namely, those at the beginning, in the midway, and at the end of each evolution phase. In other words, in the top/bottom panel of Figure 5 we combine the three top/bottom panels of Figure 4. The least-squares best fits (solid lines) yield the following relations between Class $0\left(\tau_{\mathrm{C} 0}\right)$ and Class I $\left(\tau_{\mathrm{CI}}\right)$ lifetimes (in Myr), from one hand, and the corre- sponding stellar masses (in $M_{\odot}$ ), from the other hand

$$
\begin{aligned}
& \tau_{\mathrm{C} 0}=0.18 M_{*, \mathrm{C} 0}^{0.8 \pm 0.05}, \\
& \tau_{\mathrm{CI}}=0.30 M_{*, \mathrm{CI}}^{1.2 \pm 0.05} .
\end{aligned}
$$

It is evident that a somewhat steeper than linear correlation between $\tau_{\mathrm{CI}}$ and $M_{*, \mathrm{CI}}$ persists irrespective of the stellar age, i.e., independent of whether we measure stellar masses in the beginning, in the midway, or at the end of the Class I phase. This is a direct consequence of the fact that a substantial fraction of the final stellar mass has already been accumulated by the beginning of the Class I phase and the subsequent increase in $M_{*, \mathrm{CI}}$ is not significant (see Figure 3). On the other hand, the Class 0 lifetimes demonstrate a noticeably weaker correlation with stellar masses. This is because the latter vary substantially during the Class 0 phase, which effectively spreads stellar masses along the horizontal axis in Figure 5, thus lowering the exponent in the least-squares fit.

This correlation between lifetimes and stellar masses suggests that the disagreement between the observationally inferred Class 0 and Class I lifetimes of YSOs may in part be due to variations in the initial mass function or due to observational biases toward a particular mass band. To illustrate this idea, we calculate mean lifetimes for objects in our entire mass band $\left(0.008-0.85 M_{\odot}\right.$ and 0.03-1.24 $M_{\odot}$ for Class 0 and Class I objects, respectively) and for objects in the truncated mass band (0.2$0.85 M_{\odot}$ and $0.2-1.24 M_{\odot}$ for Class 0 and Class I objects, respectively), with the latter case being confined to intermediate and upper-mass stars only. The resultant mean lifetimes of the Class 0 objects are $\left\langle\tau_{\mathrm{C} 0}\right\rangle=0.044 \mathrm{Myr}$ 


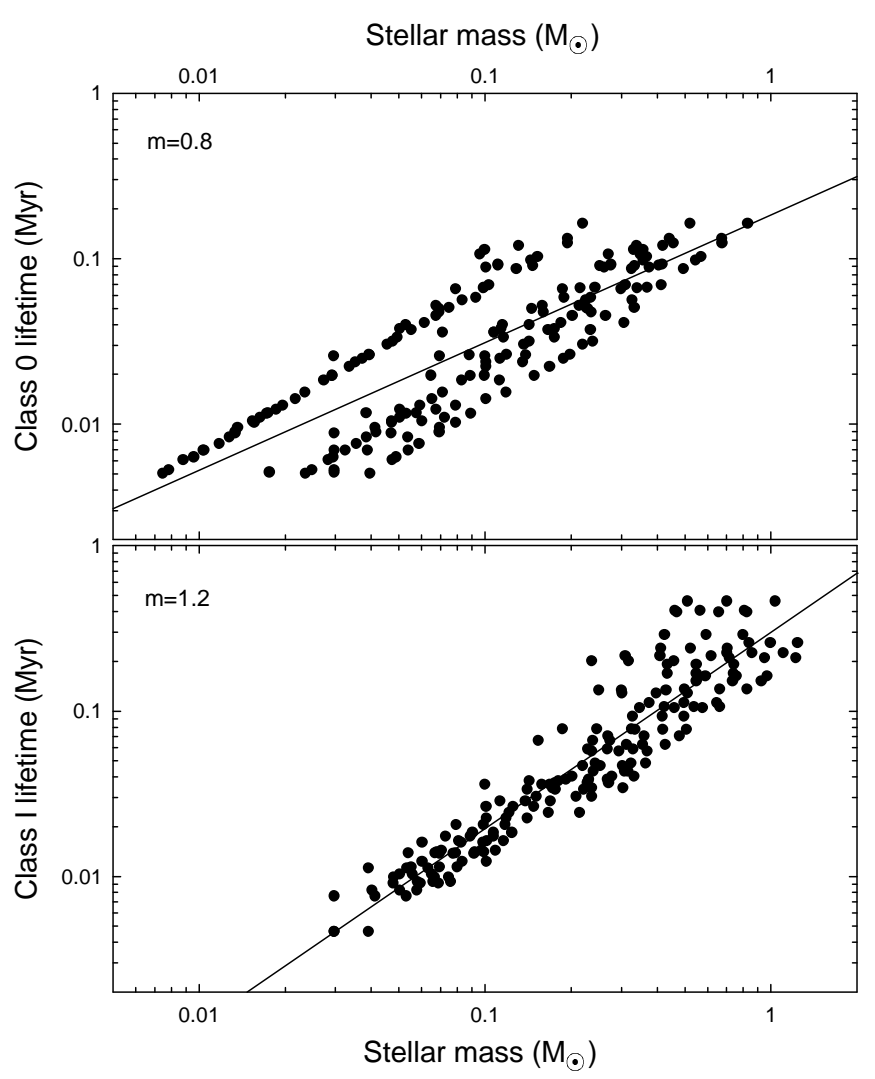

FIG. 5.- Lifetimes of the Class 0 phase (top) and Class I phase (bottom) versus stellar masses, the latter including those calculated at the beginning, in the midway, and at the end of each evolution phase. The top/bottom panels of the figure are obtained by combining the top/bottom rows of Figure 4 Solid lines present the least-squares best fits with exponents $m$.

and $\left\langle\tau_{\mathrm{C} 0}\right\rangle_{\mathrm{tr}}=0.086 \mathrm{Myr}$, respectively. In the case of the Class I objects, we obtain $\left\langle\tau_{\mathrm{CI}}\right\rangle=0.09 \mathrm{Myr}$ and $\left\langle\tau_{\mathrm{CI}}\right\rangle_{\mathrm{tr}}=0.15 \mathrm{Myr}$. It is evident that the neglect of the low-mass objects causes almost a factor of two overestimate of the Class $0 / \mathrm{I}$ lifetimes. In spite of the fact that $\tau_{\mathrm{C} 0}$ show a somewhat weaker correlation with $M_{*, \mathrm{C} 0}$, the effect of truncation is even greater than in the case of Class I lifetimes due to systematically smaller stellar masses in the Class 0 phase.

\subsection{The effect of varying initial conditions and magnetic fields}

In the previous section, we have considered model cores that differ in the adopted values of rotational to gravitational energy $\beta$, initial mass $M_{\mathrm{cl}}$, and radius $r_{\text {out }}$. However, cloud cores may vary in other aspects such as initial gas temperatures, truncation radii, initial density enhancements, and strengths of magnetic fields. In addition, the shape of the initial gas surface density profile may differ from the assumed scaling $\Sigma \propto r^{-1}$. It is computationally prohibitive to consider the effect of varying initial conditions on every model core of Section 5.1 . Therefore, we have chosen model set 2 as the reference set to estimate the effect that the varying initial conditions may have on the lifetime of the embedded phase.

Figure 6 shows our model lifetimes of the Class 0 phase (top panels) and Class I phase (bottom panels) versus stellar masses, the latter derived as described in the previous section, for five model sets. In particular, red circles correspond to model set 2, blue squares correspond to model set $2 \mathrm{~T}$, which is characterized by a higher initial gas temperature of the cloud cores $T=18 \mathrm{~K}$. Green triangles-up present model set $2 \mathrm{E}$, which is characterized by extended cloud cores with $r_{\text {out }} / r_{0}=12\left(r_{\text {out }}\right.$ is increased). Pink diamonds show model set $2 \mathrm{~A}$, in which cloud cores are characterized by the initial density enhancement amplitude $A=8$, and yellow triangles-down correspond to model set $2 \mathrm{MF}$ with the flux-to-mass ratio $\alpha_{\mathrm{m}}=0.3$. Finally, plus signs present model set $2 \mathrm{C}$, which comprises models with spatially constant surface density and angular velocity distributions. The latter are obtained using the parameters of the reference model set and setting $\Sigma=\Sigma\left(r=r_{\text {out }}\right)$ and $\Omega=\Omega\left(r=r_{\text {out }}\right)$ in Equations (6) and (7), respectively. In order words, surface densities and angular velocities in model set $2 \mathrm{C}$ are set equal to those at the boundary in the corresponding models of the reference set. The ratio $\beta=2.3 \times 10^{-3}$ is the same for all models in this section in order to exclude a mild dependence of the lifetimes on $\beta$ discussed in Section 5.1

It is evident that the varying initial conditions may have a profound effect on the duration of the embedded phase. In particular, higher initial gas temperatures and density enhancement amplitudes reduce the resulting Class 0/I lifetimes by factors of $2.5-5.5$ for objects with equal mass $M_{*}$. This is not surprising, since both effects are expected to increase the mass accretion rate from the envelope $\dot{M}_{\text {env }}$, thus shortening Class 0/I lifetimes. In the standard model of inside-out collapse, $\dot{M}_{\text {env }}$ is proportional to the cube of the sound speed $c_{\mathrm{s}}^{3}$ and to the density enhancement amplitude $A$ (Shu 1977). In our case, the increase in gas temperature is $\triangle T=1.8$ and $\dot{M}_{\text {env }}$ is expected to increase by a factor of $1.8^{3 / 2}=2.4$. Indeed, top panels in Figure 6] indicate that the corresponding Class 0 and Class I lifetimes (blue squares) have decreased on average by factors of 2.5 and 3.2 , respectively, as compared to the reference set of models (red circles). A somewhat greater drop in $\tau_{\mathrm{CI}}$ than expected indicates that the envelope empties in the late embedded phase faster than predicted by the standard model. This could be due to the influence of the cloud core's finite outer boundary and an inward-propagating rarefaction wave (cloud cores in the standard model are infinite in size). This effect becomes even more pronounced in the case of a greater initial density enhancement $A=8$ (as opposed to $A=2$ in the reference set). The corresponding Class 0 and Class I lifetimes, shown by the pink diamonds in Figure 6. have dropped by factors of 2.6 and 5.5 , respectively.

On the other hand, YSOs formed from cloud cores with greater truncation radii $r_{\text {out }} / r_{0}=12$ (green triangles-up in Figure 6) do not seem to have significantly different lifetimes as compared to those of the reference model set with $r_{\text {out }} / r_{0}=6$. This may seem counterintuitive. Indeed, more extended cores have a greater mass reservoir and are supposed to deposit their material onto a star/disk system for a longer time. However, one has to keep in mind that they would also form more massive stars. As a result, the lifetimes simply shift toward the upper-right corner in every panel of Figure 6.

YSOs formed from cloud cores with frozen-in magnetic fields $\left(\alpha_{\mathrm{m}}=0.3\right)$ reveal a complicated behavior. On one hand, their Class 0 lifetimes, as shown by yellow 


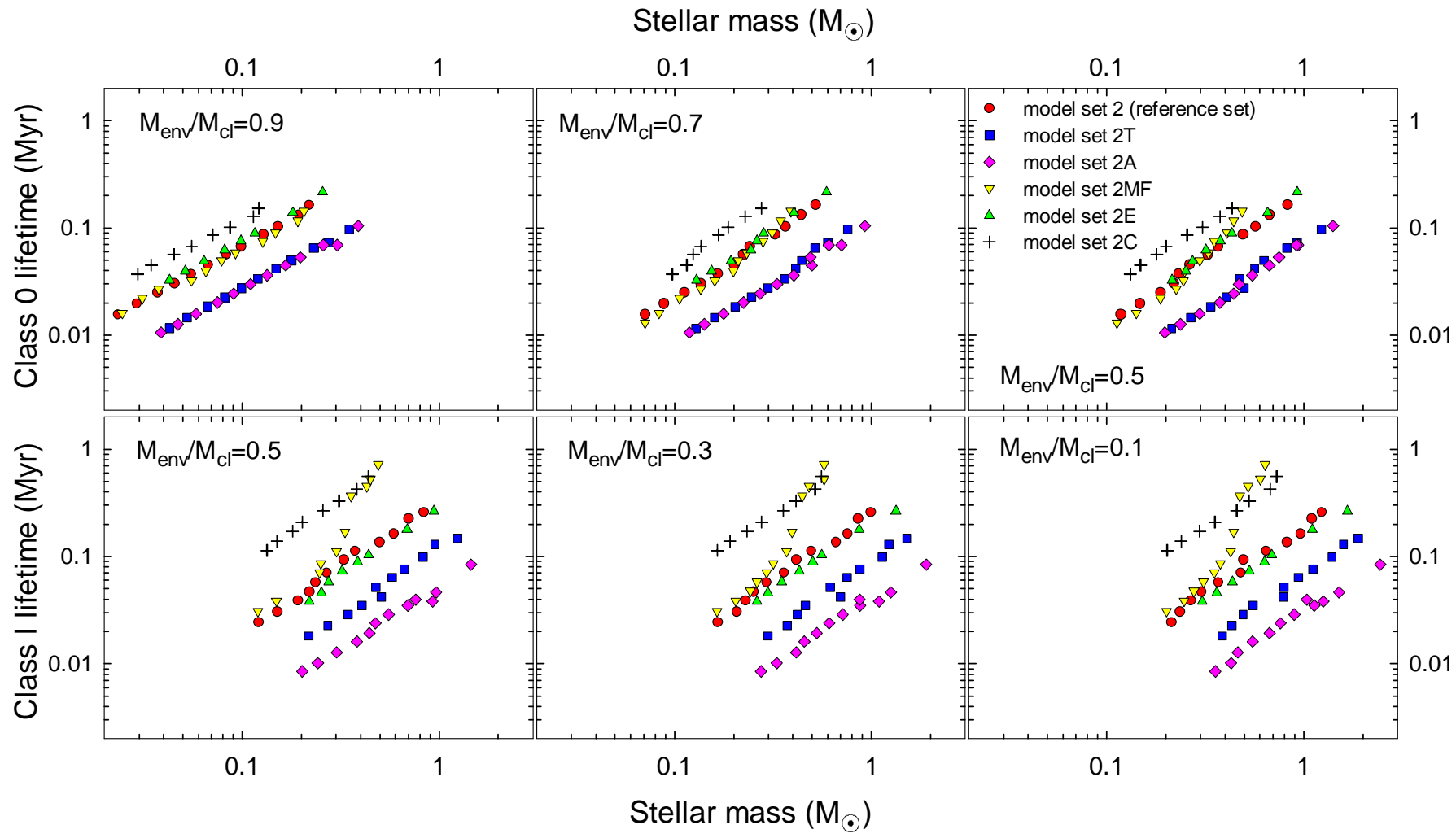

Fig. 6. - Lifetimes of the Class 0 phase (top row) and Class I phase (bottom row) versus stellar masses calculated at the beginning (right column), in the midway (middle column), and at the end (right column) of each evolution phase. The data for six model sets with varying initial conditions (see Table 1 and the text) are shown. The ratios $M_{\mathrm{env}} / M_{\mathrm{cl}}$ of the envelope mass to the initial cloud core mass are indicated in every panel.

triangles-down in the top-left panel of Figure 6, do not seem to be significantly affected. On the other hand, Class I lifetimes of the intermediate- and upper mass stars increase considerably as compared to the reference set of model. The reason why these stars are more affected than their lower-mass counterparts lies in the way that the frozen-in magnetic fields operate in cloud cores. Let us consider the outermost layer of gas. As it moves toward the center, magnetic tension increases and decelerates the collapse, prolonging the embedded phase. Magnetic field lines are attached at infinity to the natal giant molecular cloud, which evolves on a much slower time scale than the collapsing cloud core. This effectively means that the field lines are motionless at infinity. It now follows that magnetic tension would be stronger for larger (and hence more massive) cores, as it takes a larger distance for the outermost layer to travel toward the disk surface. If we recall that more massive cores produce more massive stars, a steeper dependence of $\tau_{\mathrm{CI}}$ on $M_{*, \mathrm{CI}}$ becomes evident. We note that this effect may be either magnified if higher values of $\alpha_{\mathrm{m}}$ are present in cloud cores or moderated for lower values of $\alpha_{\mathrm{m}}$. In addition, ambipolar diffusion and magnetic breaking, not included in the present numerical simulations, are expected to decrease the effect.

Finally, the initial distribution of gas surface density and angular velocity in cloud cores may also affect the resulting lifetimes. For the case of cloud cores with initially constant $\Sigma$ and $\Omega$ (plus signs in Figure 6), the resulting lifetimes are greater than those in the reference model set, the latter having both $\Sigma$ and $\Omega$ inverse proportional to radius at large radii. The increase is particularly large in the case of Class I lifetimes and is caused by a steep radial gas surface density distribution that develops in the late phases of cloud core collapse. Although cloud cores in model set $2 \mathrm{C}$ start with constant $\Sigma$, they quickly evolve into a configuration that is characterized by a gas surface density profile that declines with radius steeper than $r^{-1}$ at large radii. This acts to lower the mass infall rate from the envelope $\dot{M}=-2 \pi r v_{\mathrm{r}} \Sigma$ as compared to that in the reference model, the latter being characterized by $\Sigma \propto r^{-1}$ at $r>>r_{0}$. The effect is particularly strong in the late evolution when gas layers initially located at large radial distances begin to fall in onto the disk.

How do varying initial conditions affect the correlation between Class 0/I lifetimes and masses of the central object discussed in the previous section? To quantitatively answer this question would require to run a prohibitively large number of models for every model cloud core with all possible initial conditions. However, based on the behaviour of model cores considered in this section, we can assume that the correlations given by Equations (10) and (11) are unlikely to break completely. Indeed, in most model sets with varying initial conditions (apart from that with non-zero frozen-in magnetic fields) the slope of the correlation line is similar to that of model set 2 . It is thus the spread in the lifetimes that can affect somewhat the resulting correlation. Just to estimated the effect, we have combined Class 0 and Class I data from all models into two separate $\tau-M_{*}$ plots for each phase and found that the resulting least-squares exponents in Equations (10) and (11) decrease by as much as 0.3 . 


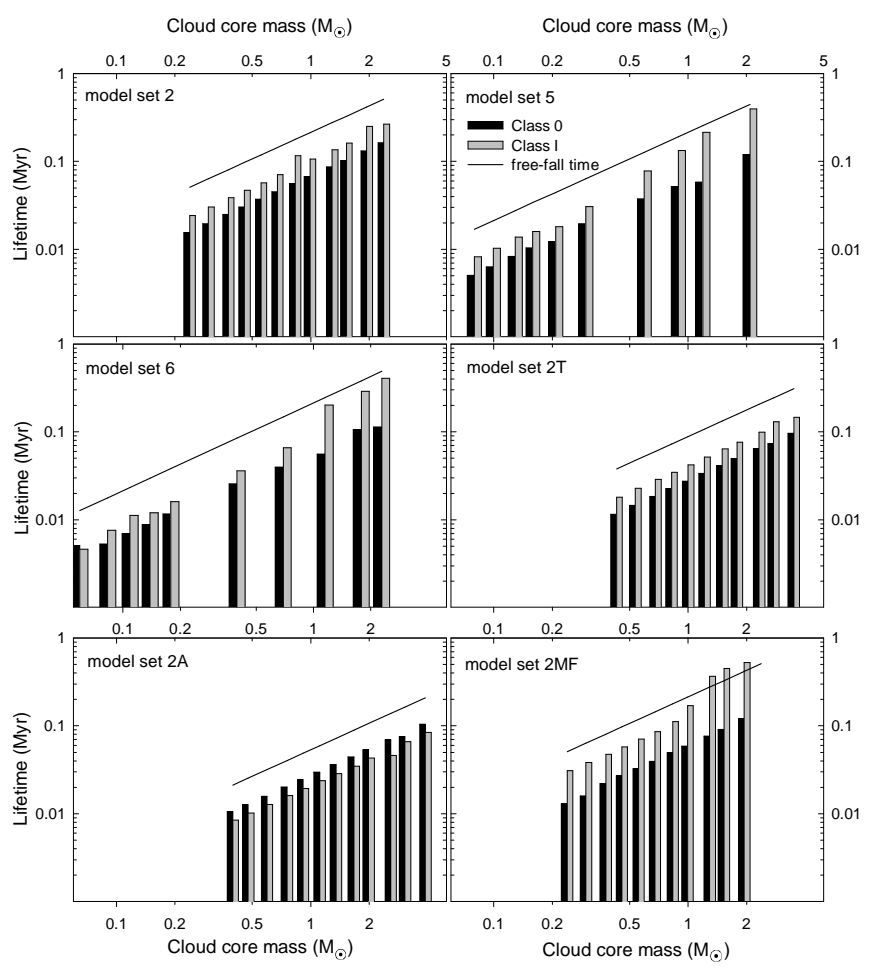

FIG. 7.-Class 0 (black bars) and Class I (grey bars) lifetimes versus initial cloud core masses for six model sets as specified in each panel. Solid lines show the free-fall time $\tau_{\mathrm{ff}}$,out of the outermost gas layer as derived from Equation A3.

\subsection{Class I lifetime versus Class 0 lifetime}

It is often useful to know how Class 0 and Class I lifetimes are related to each other for a particular YSO. It is difficult to extract such information directly from Figures 4 6 and Equations (10) and (11) are not useful because they provide data averaged over all model cores. In order to analyse the relative lifetimes, we have to choose some characteristic of a YSO in order to present $\tau_{\mathrm{C} 0}$ and $\tau_{\mathrm{CI}}$ as a function of this characteristic. We use the initial cloud core masses with a practical purpose to verify if the lifetimes indeed scale linearly with $M_{\mathrm{cl}}$, as was suggested on theoretical grounds at the beginning of Section 5

Figure 7 presents the Class 0 (black bars) and Class I (grey bars) lifetimes as a function of the initial cloud core mass $M_{\mathrm{cl}}$ for model cores from six model sets as specified in each panel. Every pair of bars represents an individual YSO formed from a cloud core with mass $M_{\mathrm{cl}}$. It is evident that $\tau_{\mathrm{CI}}$ are systematically greater than $\tau_{\mathrm{C} 0}$, except for model set $2 \mathrm{~A}$ for which $\tau_{\mathrm{CI}}<\tau_{\mathrm{C} 0}$. We note that the latter case is characterized by a rather large initial density enhancement $A=8$ and need specific conditions (such as strong shock waves) to be realized. It is also seen that the ratio $\tau_{\mathrm{CI}} / \tau_{\mathrm{C} 0}$ varies little with $M_{\mathrm{cl}}$, with a notable exception for models with magnetic fields (model set $2 \mathrm{MF}$ ) and for models with large rotation rates (model set 5 and 6 ). We have already discussed above the case with magnetic fields. As to the models with greater values of the rotational to gravitational energy $\beta$, they have more centrifugal support against gravity, which effectively prolongs the Class I phase in the upper-mass (and spatially extended) cloud cores. Excluding model set $2 \mathrm{~A}$ and the upper mass models in model sets 5,6 , and $2 \mathrm{MF}$, the mean ratio of the Class I to the Class 0 lifetime is $\tau_{\mathrm{CI}} / \tau_{\mathrm{C} 0} \approx 1.5-2.0$. For the aforementioned upper-mass models, the corresponding ratio may be as high as 3.04.0 .

The solid lines in Figure 7 show the free-fall time $\tau_{\mathrm{ff}, \text { out }}$ of the cloud's outermost layer as a function of $M_{\mathrm{cl}}$. The free-fall time is linearly proportional to $M_{\mathrm{cl}}$ (see equation (A3) in the Appendix). By comparing lifetimes with the free-fall time, one can notice that both the Class 0 and Class I lifetimes in model sets 2, 2T, and $2 \mathrm{~A}$ are also linearly proportional to $M_{\mathrm{cl}}$, as was anticipated from simple theoretical grounds in Section 5. However, the Class I lifetimes of the upper-mass model cores in model sets 5 , 6 , and $2 \mathrm{MF}$ show a significant deviation from the linear scaling typical for the low- and intermediate-mass models. This effect was not envisioned from simple theoretical considerations.

\section{ENVELOPE DEPLETION RATES}

In semi-analytic models of cloud core collapse and star/disk formation, it is often needed to know the rate at which the envelope material is accreted onto the star/disk system. We determine this rate by calculating the envelope depletion rate $\dot{M}_{\text {env }}=$ $-\left(M_{\text {env }}(t+\triangle t)-M_{\text {env }}(t)\right) / \triangle t$, with the time step $\triangle t$ set to $10^{3} \mathrm{yr}$. In the earliest stages of evolution before the disk formation, $\dot{M}_{\text {env }}$ would represent the mass accretion rate onto the star, but when the disk forms $\dot{M}_{\text {env }}$ would represent the mass accretion rate onto the disk.

The solid lines in Figure 8 show the envelope depletion rate versus time elapsed since the beginning of numerical simulations for the same four representative models as in Figure 3. The vertical dotted lines mark the onset of the Class I (left line) and Class II (right line) phases. The time evolution of $\dot{M}_{\text {env }}$ can be split into two distinct modes: a shorter period of near-constant depletion rate and a longer period of gradual (and terminal) decline of $\dot{M}_{\text {env }}$. It is seen that the boundary between these two modes lies near the vertical dotted line that separates the Class 0 and Class I phases of star formation in every model. A similar behavior of $\dot{M}_{\text {env }}$ was reported by Vorobyov \& Basu (2005) for the case of the spherically symmetric collapse of truncated cloud cores.

In the first mode, the accretion process is conceptually similar to that described by the single isothermal sphere solution (Shu 1977), wherein the material is falling freely onto the star/disk system and the accretion rate is constant and proportional to $c_{\mathrm{s}}^{3} / G$. The second mode ensues when a rarefaction wave propagating inward from the core's outer boundary hits the disk surface. From this moment on, the accretion process is no more selfsimilar, the radial gas density profile starts to steepen and the ongoing depletion of gas in the envelope causes a gradual (and terminal) decline of $\dot{M}_{\text {env }}$.

We note that $\dot{M}_{\text {env }}$ may exhibit some short-time fluctuations in the second mode due to temporary disk expansions and contractions caused by disk radial pulsations ${ }^{4}$. When expanding, the disk delivers part of its material to the envelope, thus temporarily decreasing the envelope depletion rate $\dot{M}_{\text {env }}$. The fluctuation amplitudes grow along the line of increasing cloud core masses (and, con-

4 The animation of this process can be viewed at www.astro.uwo.ca/ vorobyov (animations: burst mode of accretion) 


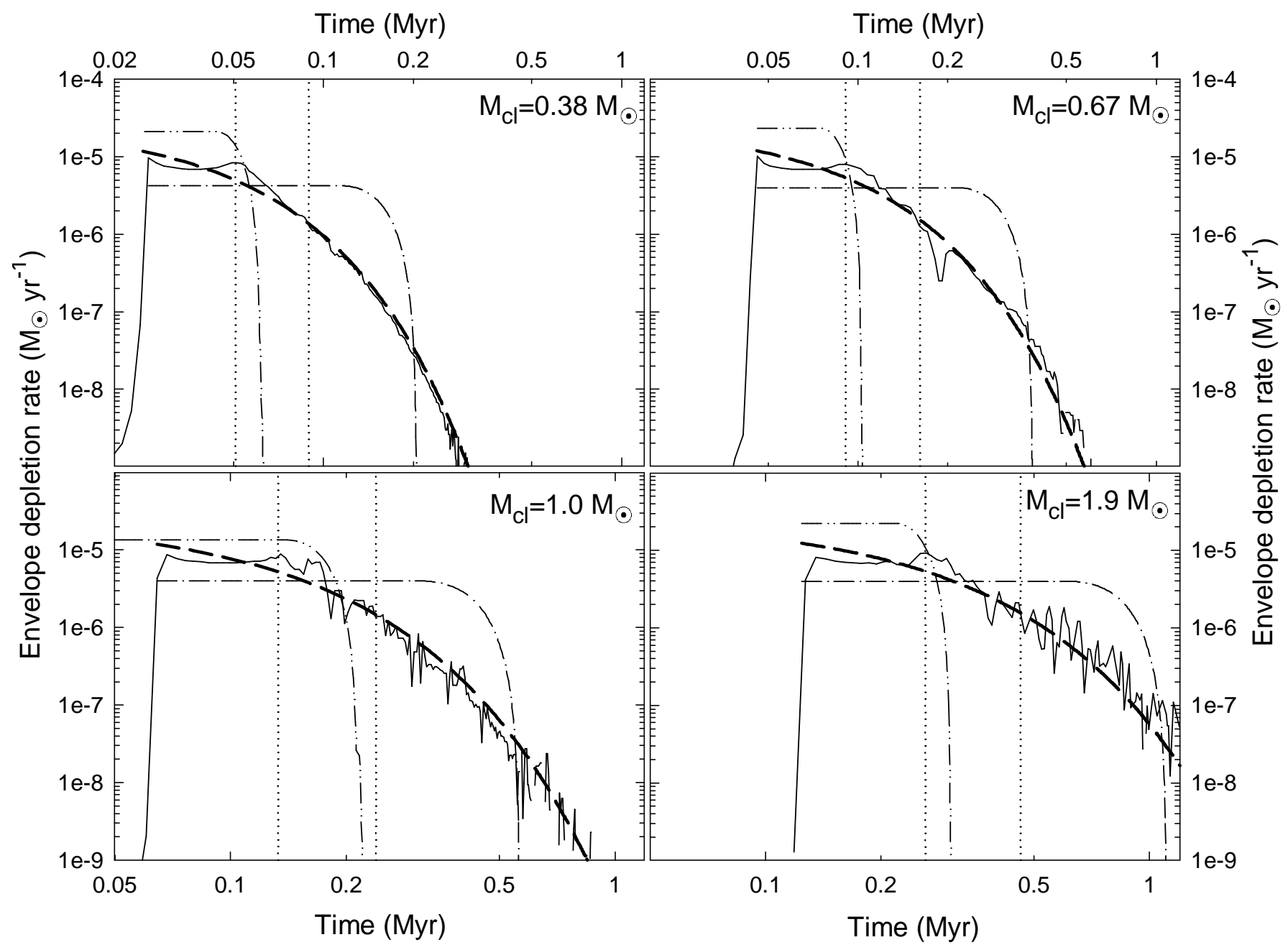

Fig. 8.- Envelope depletion rates $\dot{M}_{\text {env }}$ (or, equivalently, mass accretion rates onto a star/disk system) versus time elapsed since the beginning of numerical simulations for four representative models from model set 2 . The corresponding cloud core masses are shown in each panel. The solid lines show the model data, while the thick dashed lines are derived using Equation (14). The dashed-dotted and dash-dot-dotted lines show $\dot{M}_{\text {env }}$ as derived using the RMA function (13) with $\tau_{\mathrm{ff}}=\tau_{\mathrm{ff}, \text { out }}$ and $\tau_{\mathrm{ff}}=\tau_{\mathrm{ff}, 2 / 3}$, respectively (see the text for details). The vertical dotted lines mark the onset of the Class I (left) and Class II (right) phases.

sequently, increasing disk masses), indicating that gravitational instability lies behind these fluctuations. These fluctuations, though having a conceptually different nature, are similar in behavior to the spasmodic accretion phenomenon reported by Tassis \& Mouschovias (2005).

We now want to find a simple and convenient way to approximate our model envelope depletion rates. We consider two different functions: the first one proposed by Bontemps et al. (1996) to account for the observed outflow energetics in the embedded phase (hereafter, the BATC function) and the second one employed by Rice et al. (2009) to mimic the mass infall rates onto the star/disk system in their one-dimensional global simulations of cloud core collapse (hereafter, the RMA function)

$$
\begin{gathered}
\dot{M}_{\mathrm{BATC}}(t)=\frac{M_{\mathrm{cl}}}{\tau_{\mathrm{c}}} \times e^{-t / \tau_{\mathrm{c}}} \\
\dot{M}_{\mathrm{RMA}}(t)= \begin{cases}\frac{M_{\mathrm{cl}}}{\tau_{\mathrm{ff}}} & 0 \leq t \leq \tau_{\mathrm{ff}} \\
\frac{1}{2} \frac{M_{\mathrm{cl}}}{\tau_{\mathrm{ff}}}\left[1+\cos \frac{\pi\left(t-\tau_{\mathrm{ff}}\right)}{\tau_{\mathrm{ff}}}\right] & \tau_{\mathrm{ff}} \leq t \leq 2 \tau_{\mathrm{ff}} \\
0 & 2 \tau_{\mathrm{ff}} \leq t .\end{cases}
\end{gathered}
$$

Here, $\tau_{\mathrm{c}}$ is some characteristic time, the value of which was constrained by Bontemps et al. (1996) using the inferred duration of the embedded phase, and $\tau_{\mathrm{ff}}$ is the free-fall time.

First, we check the utility of the BATC function. When one undertakes modeling of the gravitational collapse of a cloud core, the value of $\tau_{\mathrm{c}}$ is not known a priori. Therefore, it is desirable to link $\tau_{\mathrm{c}}$ with the free-fall time $\tau_{\mathrm{ff}}=(3 \pi / 32 G \rho)^{1 / 2}$. We have experimented with various values of $\tau_{\mathrm{ff}}$, depending on the actual value of $\rho$, and recommend the following relation $\tau_{\mathrm{c}}=\tau_{\mathrm{ff} \text {,out }} / 3$, where $\tau_{\mathrm{ff} \text {,out }}$ is the free-fall time of the outermost layer derived in the Appendix. The time $t$ in Eqs. (12) and (13) is counted from the formation of the protostar (the accretion rate is negligible at the earlier time). The resulting mass accretion rate $\dot{M}_{\text {BATC }}$ is shown in Figure 8 by the thick dashed lines. It is evident that the BATC function provides an adequate fit to our model accretion rates (solid lines). This result is robust and holds for models with different values of $\beta$ and with different initial conditions.

We now proceed with considering the utility of the RMA function. Since our cloud cores are initially nonuniform, we have considered several values of $\tau_{\mathrm{ff}}$, depending on the actual value of $\rho$. The dash-dotted 
and dash-dot-dotted lines in Figure 8 show the resulting mass accretion rate $\dot{M}_{\mathrm{RMA}}$ for $\tau_{\mathrm{ff}}=\tau_{\mathrm{ff}, \text { out }}$ and $\tau_{\mathrm{ff}}=\tau_{\mathrm{ff}, 2 / 3}=\left(3 \pi /\left(32 G \rho_{2 / 3}\right)\right)^{1 / 2}$, respectively, where the latter value is the free-fall time of a layer located at $r=2 r_{\text {out }} / 3$ (i.e. at a radial distance equal to $2 / 3$ that of the cloud core outer radius) and $\rho_{2 / 3}$ is the corresponding gas volume density. It is obvious that, regardless of the value of $\tau_{\mathrm{ff}}$, the RMA function fails to provide an acceptable fit to our model accretion rates (solid lines). To summarize, we suggest using the following equation as the mass accretion rate onto a star/disk system

$$
\dot{M}_{\text {env }}(t)=\frac{3 M_{\mathrm{cl}}}{\tau_{\mathrm{ff}, \text { out }}} \times e^{-3 t / \tau_{\mathrm{ff}, \text { out }}},
$$

where time $t$ should be counted from the formation of a central star and $\tau_{\mathrm{ff}, \text { out }}$ (see Equation $\mathrm{A3}$ ) is the free-fall time of the outermost gas layer of a cloud core with mass $M_{\mathrm{cl}}$.

\section{CONCLUSIONS}

Using numerical hydrodynamics simulations, we have computed the gravitational collapse of a large set of cloud cores. We start from a gravitationally unstable, pre-stellar phase and terminate the simulations in the Class II phase when most of the cloud core has been accreted by the forming star/disk system. We have considered cloud cores with various initial masses $\left(M_{\mathrm{cl}}=0.06-3.9 M_{\odot}\right)$, ratios of the rotational to the gravitational energy $\left(\beta=(0.2-2.2) \times 10^{-2}\right)$, initial gas temperatures $\left(T_{0}=10-18 \mathrm{~K}\right)$, truncation radii $\left(r_{\text {out }} / r_{0}=6-12\right)$, strengths of frozen-in magnetic fields $\left(\alpha_{\mathrm{m}}=0-0.3\right)$, initial gas density enhancements $(A=2-8)$, and initial radial profiles of the gas surface density and angular velocity $\left(\Sigma, \Omega \propto r^{-1}\right.$ and $\Sigma, \Omega=$ const $)$.

We employ a sophisticated method for distinguishing between the infalling envelope and the forming disk in our numerical simulations and determine the duration of the embedded phase of star formation, adopting a classification scheme based on the remaining mass in the envelope (André et al. 1993). We also calculate the envelope depletion rate $\dot{M}_{\text {env }}$ (or, equivalently, the rate of mass accretion onto the star/disk system) and test the utility of two empirical functions for $\dot{M}_{\text {env }}$ provided by Bontemps et al. (1996) (BATC function) and Rice et al. (2009) (RMA function). We find the following.

1. Class $0\left(\tau_{\mathrm{C} 0}\right)$ and Class I $\left(\tau_{\mathrm{CI}}\right)$ lifetimes correlate with the corresponding stellar mass $M_{*, \mathrm{C} 0}$ and $M_{* \text {,CI }}$, however the scaling is more complex than can be expected from simple theoretical grounds based on the linear correlation between the freefall time and the cloud core mass. In particular, the form of the correlation depends on the cloud core properties such as the initial ratio of rotational to gravitational energy, initial gas temperature, density enhancement amplitude, and the initial radial distribution of gas surface density and angular velocity. In addition, frozen-in magnetic fields with constant flux-to-mass ratio can substantially influence the Class I lifetimes. The correlation is further complicated due to the fact that the stellar mass varies considerably during the Class 0 phase. This makes the $\tau_{\mathrm{C} 0}-M_{*, \mathrm{C} 0}$ correlation dependent on the stellar age as well.
2. When cloud cores with varying rotation rates, masses and sizes (but otherwise identical) are considered, Class 0 and Class I lifetimes have suband super-linear correlations with the corresponding stellar masses, respectively. These correlations extend from sub-stellar masses $\left(\sim 0.01 M_{\odot}\right)$ to at least solar masses $\left(\sim 1.5 M_{\odot}\right)$ and obey the following scaling laws $\tau_{\mathrm{C} 0}=0.18 M_{*, \mathrm{C} 0}^{0.8 \pm 0.05}$ and $\tau_{\mathrm{CI}}=0.3 M_{*, \mathrm{CI}}^{1.2 \pm 0.05}$ for Class $0 / \mathrm{I}$ stars of all possible ages. This makes the observational determination of the mean lifetimes sensitive to the form of the initial mass function and/or to instrumental biases toward a particular mass band. For instance, our modeling predicts a mean Class 0 lifetime of $\left\langle\tau_{\mathrm{C} 0}\right\rangle=0.044 \mathrm{Myr}$ for stars in the 0.008$0.85 M_{\odot}$ mass range and $\left\langle\tau_{\mathrm{C} 0, \mathrm{tr}}\right\rangle=0.086 \mathrm{Myr}$ for stars in the $0.02-0.85 M_{\odot}$ range. In the case of the Class I objects, we obtain $\left\langle\tau_{\mathrm{CI}}\right\rangle=0.09 \mathrm{Myr}$ and $\left\langle\tau_{\mathrm{CI}}\right\rangle_{\mathrm{tr}}=0.15 \mathrm{Myr}$ for stars in the 0.03-1.24 $M_{\odot}$ and $0.2-1.24 M_{\odot}$ mass ranges, respectively. It is evident that the neglect of objects at the lower mass end results in almost a factor of 2 overestimate of the mean lifetimes.

3. An increase in the initial cloud core temperature and density enhancement amplitude tend to lower the Class 0 and Class I lifetimes, whereas cloud cores with initially constant gas surface density and angular velocity distributions (as compared to those with $\Sigma, \Omega \propto r^{-1}$ ) have longer Class 0 and especially Class I lifetimes. In addition, frozenin magnetic fields may increase the Class I lifetimes, particularly for the upper-mass cloud cores, thus steepening the corresponding $\tau_{\mathrm{CI}}-M_{* \text {,CI }}$ relation. The net effect of varying initial condition is to weaken the aforementioned sub-linear (Class 0) and super-linear (Class I) correlations between the lifetimes and stellar masses by decreasing the corresponding exponents by as much as 0.3 . However, more accurate modeling with ambipolar diffusion and magnetic braking taken into account is needed to accurately assess the magnitude of this effect. The outer truncation radius of a cloud core has little effect on the resulting lifetimes.

4. Most cloud cores give birth to YSOs whose Class I lifetimes are longer than those of the Class 0 phase by roughly a factor of 1.5-2.0. A notable exception are YSOs formed from cloud cores a with large initial density enhancement. In the latter case, the duration of the Class I phase may actually be shorter than that of the Class 0 . In addition, the upper-mass models $M_{\mathrm{cl}}>1 M_{\odot}$ with frozen-in magnetic fields and high cloud core rotation rates $\beta>10^{-2}$ may have the $\tau_{\mathrm{CI}} / \tau_{\mathrm{C} 0}$ ratios as large as $3.0-4.0$.

5. The time evolution of $\dot{M}_{\text {env }}$ reveals two distinct modes: a shorter period of near-constant depletion rate and a longer period of gradual (and terminal) decline of $\dot{M}_{\text {env }}$. The boundary between these two modes lies near the end of the Class 0 phase and the beginning of Class I phase. In the later mode, $\dot{M}_{\text {env }}$ may show short-term fluctuations due 
to episodic disk expansions and contractions. The BATC function may provide an adequate fit to our model envelope depletion rates if the characteristic time of decline of $\dot{M}_{\text {env }}$ is chosen properly. The RMA function fails to provide an acceptable fit to our model data, irrespective of the free parameters.

We emphasize that our lifetimes have been derived based on the AWTB classification scheme André et al. 1993) and may change by a factor of unity if other schemes are used. For instance, if we re-define the boundary between the Class I and Class II phases in the AWTB scheme and assume that the Class II phase begins when the envelope mass drops below $5 \%$ of the initial cloud core mass (in contrast to $10 \%$ adopted in our paper), the resulting Class I lifetimes in model set 2 increase by a factor of 1.5. However, the derived trends and correlations between the lifetime of the embedded phase, from one hand, and the stellar masses, from the other hand, are expect to stay valid irrespective of the classification scheme used.

The author is thankful to the anonymous referee for suggestions and comments that helped to improve the final manuscript. The author gratefully acknowledges present support from an ACEnet Fellowship and Prof. Rob Thacker. Numerical simulations were done on the Atlantic Computational Excellence Network (ACEnet) and at the Center of Collective Supercomputer Resources, Taganrog Technological Institute at South Federal University.

\section{REFERENCES}

André, P., Ward-Thompson, D., \& Barsony, M. 1993, ApJ, 406, 122

André, P., \& Montmerle, T. 1994, ApJ, 420, 837

Bacmann, A., André, P., Puget, J.-L., Abergel, A., Bontemps, S., Ward-Thompson, D., 2000, A\&A, 361, 555

Basu, S. 1997, ApJ, 485, 240

Boley, A. C., Hayfield, T., Mayer, L., \& Durisen, R. H. 2009, astro-ph/09094543

Bontemps, S., André, P., Terebey, S., Cabrit, S. 1996, A\&A, 311, 858

Caselli, P., Benson, P. J., Myers, P. C., \& Tafalla, M. 2002, 572, 238

Dapp, W. B., Basu, S. 2009, MNRAS, 395, 1092.

Dunham, M. M., Evans II, N. J., Terebey, S., Dullemond, P., Young, C. H. 2010, to appear in ApJ

Enoch, M. L., Eet al. 2006, ApJ, 638, 293

Enoch M. L., Evans II, N. J., Sargent, A. I., \& Glenn, J. 2009, ApJ, 692,973

Evans II, N. J., Dunham, M. M., Jorgensen, J. K., Enoch, M. L., Merín, B., van Dishoeck, E. F., et al. ApJSS, 181, 321

Froebrich, D., Schmeja, S., Smith, M. D., \& Klessen, R. S. 2006, MNRAS, 368, 435

Greene, T. P., Wilking, B. A., André, P., Young, E. T., \& Lada, C. J. 1994, ApJ, 434, 614

Hatchell, J., Fuller, G. A., Richer, J. S., Harries, T. J., \& Ladd E. F. 2007, A\&A, 468, 1009
Kenyon, S. J., \& Hartmann, L. 1995, ApJS, 101, 117

Kratter, K. M., Matzner, C. D., Krumholz, M. R., \& Klein, R. I., astro-ph:0907.3476

Lada, Ch. J. 1987, in IAU Symp. 115, Star Forming Regions, ed. M. Peimbert \& J. Jugaku (Dordrecht: Reidel), 1

Larson, R. B., 2003, Rep. Prog. Phys., 66, 1651

Lin, D.N.C., Pringle, J.E. 1990, ApJ 358, 515

Masunaga, H., \& Inutsuka, S. 2000, ApJ, 531, 350

Myers, P. C., \& Ladd, E. F. 1993, ApJ, 413, L47

Rice, W. K. M., Mayo, J. H., \& Armitage, P. J. 2009, astroph:0911.1202

Shu, F. H. 1977, ApJ, 214, 488

Tassis, K., \& Mouschovias, T. C. 2005, ApJ, 618, 783

Visser, A. E., Richer, J. S., Chandler, C. J. 2002, AJ, 124, 2756

Vorobyov, E. I., \& Basu, S. 2005, MNRAS, 360, 675

Vorobyov, E. I., \& Basu, S. 2006, ApJ, 650, 956

Vorobyov, E. I., 2009a, ApJ, 692, 1609

Vorobyov, E. I. 2009b, ApJ, 704, 715

Vorobyov, E. I. 2009c, New Astronomy, 15, 24

Vorobyov, E. I., \& Basu, S. 2009a, MNRAS, 393, 822

Vorobyov, E. I., \& Basu, S. 2009b, ApJ, 703, 922

Wilking, B. A., Lada, C. J., \& Young, E. T. 1989, ApJ, 340, 823

\section{APPENDIX \\ RELATION BETWEEN THE CLOUD CORE MASS AND THE FREE-FALL TIME}

If the initial gas volume density distribution is non-uniform, the usual definition of $\tau_{\mathrm{ff}}$ needs to be further clarified as to what value of $\rho$ should be used. In our case, it is convenient to set $\rho=\rho_{\text {out }}=\rho\left(r=r_{\text {out }}\right)$, i.e. we use the gas volume density at the outer cloud core boundary. Then, the resulting free-fall time of the outermost gas layer becomes $\tau_{\text {ff, out }}=\sqrt{3 \pi /\left(32 G \rho_{\text {out }}\right)}$.

We now need to relate $\tau_{\mathrm{ff}, \text { out }}$ with the initial cloud core mass $M_{\mathrm{cl}}$. Neglecting a small plateau in the gas surface density profile at $r \lesssim r_{0}$, we can write (see Section 3)

$$
\Sigma=\frac{A^{1 / 2} c_{\mathrm{s}}^{2}}{\pi G r}
$$

The mass of a cloud core with this gas surface density distribution is

$$
M_{\mathrm{cl}}=\frac{2 A^{1 / 2} c_{\mathrm{s}}^{2} r_{\mathrm{out}}}{G}=\frac{2 A c_{\mathrm{s}}^{4}}{\pi G \Sigma_{\mathrm{out}}},
$$

where $\Sigma_{\text {out }}$ is the gas surface density at the cloud core's outer boundary. Assuming a local vertical hydrostatic equilibrium with the scale height $Z=c_{\mathrm{s}}^{2} /(\pi G \Sigma)$, approximating the gas volume density as $\rho=\Sigma /(2 Z)$, and substituting the resulting value of $\rho_{\text {out }}=\Sigma_{\text {out }}^{2} \pi G /\left(2 c_{\mathrm{s}}^{2}\right)$ in the expression for $\tau_{\mathrm{ff}, \text { out }}$, we finally obtain

$$
\tau_{\mathrm{ff}, \text { out }}[\mathrm{Myr}]=\frac{3.8}{A}\left(\frac{\mu}{T_{0}}\right)^{3 / 2}\left(\frac{M_{\mathrm{cl}}}{M_{\odot}}\right),
$$

where $T_{0}$ is the initial gas temperature, $\mu$ is the mean molecular weight, and $A$ is the amplitude of initial density enhancement. 